\title{
Get Ready for 100 Years of Active Spine Life Using Percutaneous Endoscopic Spine Surgery (PESS)
}

\author{
Hyeun Sung Kim${ }^{1}$, Nitin Adsul ${ }^{1}$, Ki Joon Kim², Jee-Soo Jang ${ }^{2}$, Il-Tae Jang ${ }^{1}$, Seong-Hoon $\mathrm{Oh}^{3}$ \\ ${ }^{1}$ Department of Neurosurgery, Nanoori Gangnam Hospital, Seoul, \\ ${ }^{2}$ Department of Neurosurgery, Nanoori Suwon Hospital, Suwon, \\ ${ }^{3}$ Department of Neurosurgery, Nanoori Incheon Hospital, Incheon, Korea
}

Corresponding Author:

Nitin Adsul, MD

Department of Neurosurgery,

Nanoori Gangnam Hospital,

731, Eonju-ro, Gangnam-gu,

Seoul 06048, Korea

Tel: $+82-2-6003-9767$

Fax: +82-2-3445-9755

E-mail: no1.nitinadsul@gmail.com

Received: February 2, 2018

Revised: February 4, 2018

Accepted: February 5, 2018

\begin{abstract}
Lumbar spinal stenosis is the most common indication for spinal surgery in patients older than 65 years. After the introduction of Kambin's safety triangle, percutaneous endoscopic spine surgery has started through transforaminal approach for discectomy and is now being extended to spinal stenosis through interlaminar approach, which is an important part of the degenerative spinal disease. With the increase in human longevity, the development of effective treatment for degenerative diseases is inevitable, and future percutaneous endoscopic spine surgery (PESS) will play a very important role in maintaining the health of this 'super-aged' population. Endoscopic techniques impart minimal approach related disruption of normal spinal anatomy and function while concomitantly increasing functional visualization and correction of degenerative stenosis. Advantages of full endoscopic spine surgeries are less soft tissue dissection, less blood loss, reduced hospital admission days, early functional recovery and enhancement in the quality of life. With proper training and advancement in equipment and technologies, percutaneous endoscopic spine surgery will be able to successfully treat the aging spine.
\end{abstract}

Key Words: Percutaneous endoscopic spine surgery, Transforaminal, Interlaminar, Discectomy, Decompression

\section{INTRODUCTION}

The fields of spine surgery have experienced rapid growth over the past decade, yet little research has focused on the issues related to older age. Perioperative complications are frequently encountered in elderly patients owing to comorbid cardiovascular and renal disease, poor nutritional status and immobility. Concerns regarding perioperative complications may be allayed by decreasing the invasiveness with which the spine is approached. Smith and Fessler" have mentioned without exaggeration about the paradigm change in spine surgery accompanying the evolution of MISS (Minimally Invasive Spine Surgery) techniques. Ideal surgical approach to the spine is the one, which causes minimal approach related disruption of normal spinal anatomy and function.

Full endoscopic surgical approaches to the spine differ from other minimally invasive surgical techniques due to the unique technical qualities of the spinal working endoscope. Use of spinal endoscope facilitates the principles of minimally invasive spinal techniques by allowing the specialist to visualize spinal structures in magnified view ${ }^{2}$. This magnified view facilitates surgical treatment with minimal surgical damage to normal anatomical structures.

Initially, standard endoscopic surgical techniques were restricted to the lumbar, cervical and thoracic disc herniations ${ }^{3)}$. Recent advances in endoscopic optical innovation and surgical techniques allow the care and treatment of other spinal conditions including central and foraminal stenosis decompression of the lumbar, thoracic, and cervical spine including anterior cervical decompression ${ }^{4-7)}$.

Advantages of full endoscopic spine surgeries are less soft tissue dissection, less blood loss, reduced hospital admission days, early functional recovery and enhancement in the quality of life ${ }^{8)}$. With the increase in human longevity, the development of effective treatment for degenerative diseases is inevitable, and future percutaneous endoscopic spine surgery (PESS) will play a very important role in maintaining health of this 'Superaged' population.

\section{HISTORICAL REVIEW}

Albert E. Telfeian et al. ${ }^{9)}$ divided the history of endoscopic spine surgery into 3 phases: inspiration, invention, and innovation. The inspired early spine surgeons desired an endoscopic spine surgery for accessing lumbar disc herniations, which would 
be less invasive than conventional open surgical techniques. They approached the disc pathology through Kambin's triangle. The invention would then be required in the armamentarium to make endoscopic discectomy a feasible and then a successful procedure. With more than 50 years of nitty-gritty (Table 1), the innovators now have the inventory and learning experience to treat various spine issues besides the herniated lumbar disc prolapse for which this technique was proposed.

\section{NEED TO APPROACH THROUGH KAMBIN'S TRIANGLE}

\section{Complication of the Conventional Open Surgery}

In the literature, the incidence of spine operations has increased than before in patients of age 65 and above. Distinctively, in a 2010 report, a 28-fold rise in spine fusion surgeries was observed for elderly patients. Elderly patients with more co-morbid factors, including diabetes mellitus, hypertension, coronary artery disease (prior procedures), depression, and obesity, experience higher postoperative complication rates and expenditure ${ }^{10)}$. In literature comparing three operative methods for different age groups with degenerative scoliosis and radiculopathy with minimum 2-year follow-up ${ }^{11)}$. In patients with average age of 76.4 who underwent lumbar decompression alone, the complication rate was $10 \%$. In patients with average age of 70.4 years who underwent decompression/limited fusion (1-2 levels), the complication rate was $40 \%$. Nevertheless, for those averaging a lower 62.5 years of age with multilevel full-curve fusions, the highest complication rate of $56 \%$ was observed. In conclusion, had the average age for the latter group been higher, the complication rate would have risen even further. Notably, the less extensive procedures (decompression alone or decompression with limited fusion) yielded considerable en- hancement on the postoperative Oswestry Disability Index, while the full fusion group did not.

\section{Need of Structural Preservation Spine Surgery}

The prime purpose of improving pain and neurological deficit in the practice of spine surgery is shifting to a more ambitious goal, namely to improve the overall quality of life and the future of patients through three key measures (1) preserving the intraspinal anatomical structures; (2) preserving the paraspinal anatomical structures; and (3) preserving the functionality of the spinal segment. Thus, three new concepts have emerged (a) minimal surgery; (b) minimal access surgery; and (c) motion preservation surgery. These concepts are covered in a new term, minimally invasive spine surgery (MISS). The term "MISS" is not about one or several particular surgical techniques, but a new way of thinking, a new viewpoint and a new philosophy ${ }^{12}$. Even if the advancements in minimally invasive spine surgery are recent, its application includes all spine segments and almost all the existing conditions.

\section{Kambin's Triangle - True Minimized Option for Degenerative Lumbar Disc Herniation}

Direct access to the disc through transforaminal approach, was advanced and universalized by Prof. Anthony Yeung, and symbolize the first major accomplishment of percutaneous endoscopic discectomy. Transforaminal discectomy through posterolateral approach uses the natural anatomical window of the intervertebral foramen to minimize damage during approach. The working cannula exceeds through muscle planes, passes through the foramen and between the exiting and traversing nerve roots (Kambin's triangle) to enter directly into the disc ${ }^{13)}$. Using this approach neither cutting of muscle nor resection

Table 1. Brief history of endoscopic spine surgery

\begin{tabular}{lll}
\hline \hline Year & \multicolumn{1}{c}{ Surgeon } & \multicolumn{1}{c}{ Contribution } \\
\hline 1963 & Lymen Smith et al. & Percutaneous Chemonucleolysis \\
1970 Kambin & Mechanical nuclear debulking by inserting Craig cannula using posterolateral approach \\
1985 Onik et al. & Central nucleotomy using nucleotome \\
1990 Kambin & Described the boundaries of safe working zone for posterolateral approach \\
1996 Mathews & Transforaminal endoscopic discectomy through foramen \\
1991 Kambin and Sampson & Developed cannula (10-23 mm ID) for interlaminar and transforaminal endoscopy \\
1997 Anthony Yeung & Designed YESS endoscope \\
2007 Lee SC et al. & Half and Half technique and epiduroscopic approach \\
2007 Ruetten et al. & Percutaneous endoscopic Interlaminar discectomy \\
2008 Choi G et al. & Foraminoplastic technique for highly migrated intracanal disc herniations \\
2009 & Kim HS et al. & Suprapedicular approach and percutaneous endoscopic stenosis lumbar decompression \\
& & (PESLD) \\
2011 & Yeom KS et al. and Kim JS et al. & Contralateral approach \\
2017 & Kim HS et al. & Percutaneous full endoscopic bilateral lumbar decompression of spinal stenosis through \\
& & uniportal-contralateral approach \\
\hline
\end{tabular}


of bone or ligament is required during discectomy.

The transforaminal approach provides direct access to the disc for nuclear decompression and annuloplasty. After withdrawing the working cannula from the disc, structures outside the disc can be easily accessed for foraminoplasty or for removing a sequestered disc fragment. Additionally, by changing the skin entry point and the angle of approach, different disc levels can be accessed using the same incision ${ }^{14)}$.

\section{INSIDE OUT APPROACH AND OUTSIDE IN APPROACH}

Depending on how the pathology is approached, posterolateral techniques can be classified into "inside-out" and "outside-in" techniques (Table 2). The inside-out techniques such as the YESS technique starts working within the disc ${ }^{15,16)}$. If needed, the working system can be repositioned so that the tip of the working cannula is located in the foramen or the spinal canal. The inside-out techniques are suitable for treating internal disc disruption, disc tears and bulges, and pathologies located in the foramen or spinal canal. But for minor disc pathologies, especially when the pathology is not located within the disc, inside-out techniques may cause too much damage by removing normal disc tissue.

For the outside-in techniques, the opening of the working cannula is initially positioned in the foramen or epidural space and then pushed to the inside the target disc, if needed ${ }^{17,18)}$. The outside-in techniques work well when treating foraminal disc herniations and extraforaminal disc herniations ${ }^{19)}$. But the technique may demand an aggressive undercutting of the facet with drill tools to reach centrally located disc herniations at L5-S1, posing a risk of damaging the nerves and dura. For both inside-out and outside-in techniques, it is challenging to treat spinal pathologies located at the L5-S1 level, especially in male patients because of the interference of the iliac crest.

\section{APPROACH: LESS ANGLED OR POSTEROLATERAL}

As compared to posterior lateral approach, the less angled extreme lateral approach has following advantages.
1. It approaches the epidural space directly; cutting of normal annulus fibrosus is not required ${ }^{20)}$. The cutting of annulus fibrosus causes severe pain during surgery and persistent pain after surgery. Moreover, this is one of the leading causes of recurrence after surgery.

2. It allows excision of central disc herniations by approaching in parallel with the posterior longitudinal ligament.

3. As this approach uses epidural space which provides an increased vertical range to manipulate the endoscope than the posterior lateral approach and allows excision of high migrated disc herniations ${ }^{21)}$.

However, the less angled approach has the potential for visceral damage and to some extent the possibility of exiting nerve root injuries so this approach is not used widely at present. Additionally, the less angled approach can be used effectively with the lever technique ${ }^{22)}$ or outside in procedure and it must be considered in terms of procedure safety.

\section{TECHNICAL CONSIDERATION OF PERCUTANEOUS ENDOSCOPIC SPINE SURGERY}

\section{Three Different Approach Routes}

Since the conventional inside-out technique approaches only the intervertebral route, it is difficult to remove the disc if it is migrated superiorly or inferiorly. However, there are three different routes in the transforaminal space anatomically, and if used effectively, percutaneous endoscopic spine surgery can be applied to a wider range of lumbar disc herniation.

Central, paracentral, and high canal compromised LDH were approached by the intervertebral route; foraminal, superiorly migrated and far lateral LDH were approached by a foraminal route (Fig. 1) and inferiorly migrated LDH was approached by suprapedicular route ${ }^{23)}$.

\section{Overcome the Anatomical Limitation using the Evolution of the Endoscopic Drill}

One of the main anatomical barriers of the transforaminal

Table 2. Difference between Inside out and outside in technique of endoscopy

\begin{tabular}{|c|c|c|}
\hline & Inside out & Outside in \\
\hline Method & $\begin{array}{l}\text { - Insert the working channel directly into the intradiscal } \\
\text { space after annulus fenestration with fluoroscopic } \\
\text { guide. Then, change the working channel target } \\
\text { toward the disc fragment. }\end{array}$ & $\begin{array}{l}\text { - Insert the working channel into the transforaminal } \\
\text { space before fenestration of the annulus. Then, } \\
\text { change the working channel target towards the } \\
\text { disc fragment. }\end{array}$ \\
\hline Advantages & $\begin{array}{l}\text { - Easy application } \\
\text { - Direct contact with the target point }\end{array}$ & $\begin{array}{l}\text { - Preserves normal anatomical structures of disc } \\
\text { - Easy accessibility to target point } \\
\text { - Applicable for all types of disc herniations }\end{array}$ \\
\hline Disadvantages & $\begin{array}{l}\text { - Painful: Annulus fenestration. } \\
\text { - Distortion: Anatomical structures } \\
\text { - Limited indication }\end{array}$ & $\begin{array}{l}\text { - Learning curve } \\
\text { - Movable working cannula can give neural or } \\
\text { structural damage }\end{array}$ \\
\hline
\end{tabular}


area is the bony structures. In recent years, the developments in endoscopic drill have solved this problem. The use of a highspeed drill under clear endoscopic visualization facilitates safer and more efficient bone removal (Fig. 2). With the articulating bone burr, we can change the drilling direction and cover up more working area. After the undercutting of the hypertrophied facet and part of the pedicle, the remaining bony work and soft tissues can be cleared using endoscopic punches, forceps, and $a$ laser $^{24)}$.

\section{Transforaminal Approach Vs Interlaminar Approach}

The conventional percutaneous endoscopic interlaminar approach is similar to microscope-assisted surgery because of removal of ligamentum flavum and cutting of annulus. Therefore, the transforaminal approach has been preferred in terms of minimizing invasive surgery. Although many techniques of PELD (percutaneous endoscopic lumbar discectomy) have been introduced, most studies at the L5-S1 level have preferred the interlaminar approach ${ }^{25,26)}$. It is thought that the high iliac crest, narrow foramen, and a large facet joint are a barrier to performing transforaminal PELD. On the other hand, Yeung and Tsou ${ }^{16)}$ suggested that PELD could access all lumbar levels, even L5S1. The favorable approach for L5-S1 level by endoscopic route has been a matter of debate for long. However, a surgeons preference for the interlaminar or transforaminal route, in addition to the height of iliac bone continue to remain an important factor in this decision.

\section{Importance of Interlaminar Approach}

Percutaneous endoscopy lumbar discectomy has an anatomi-
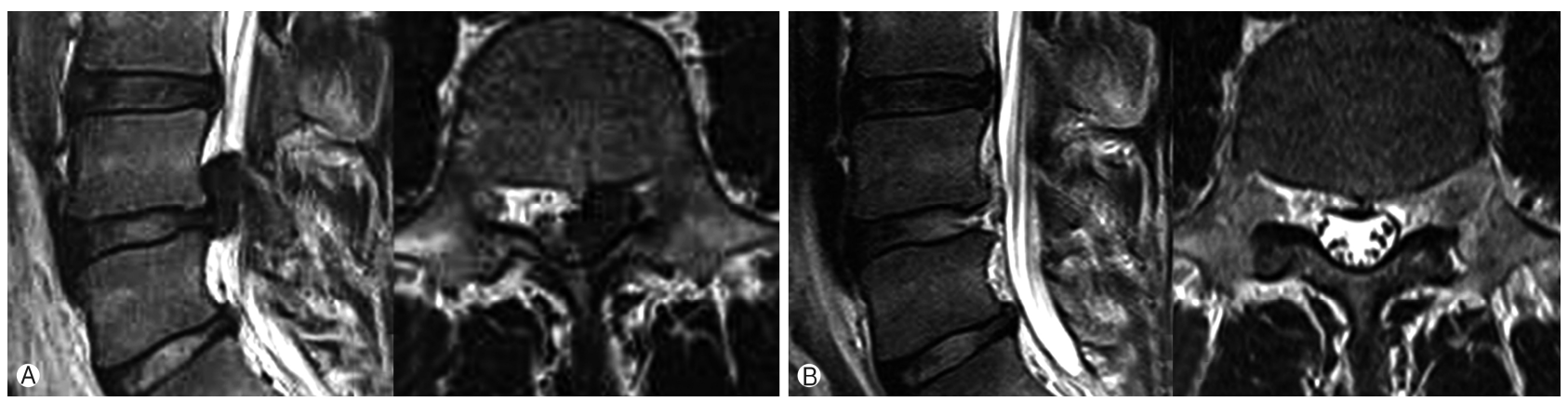

Fig. 1. Three different routes help in effectively removing even the most disc protrusions. Forty-one years old male with highly superior migrated L4-5 disc: (A) Preoperative MRI, (B) Postoperative MRI 4 hours after surgery.

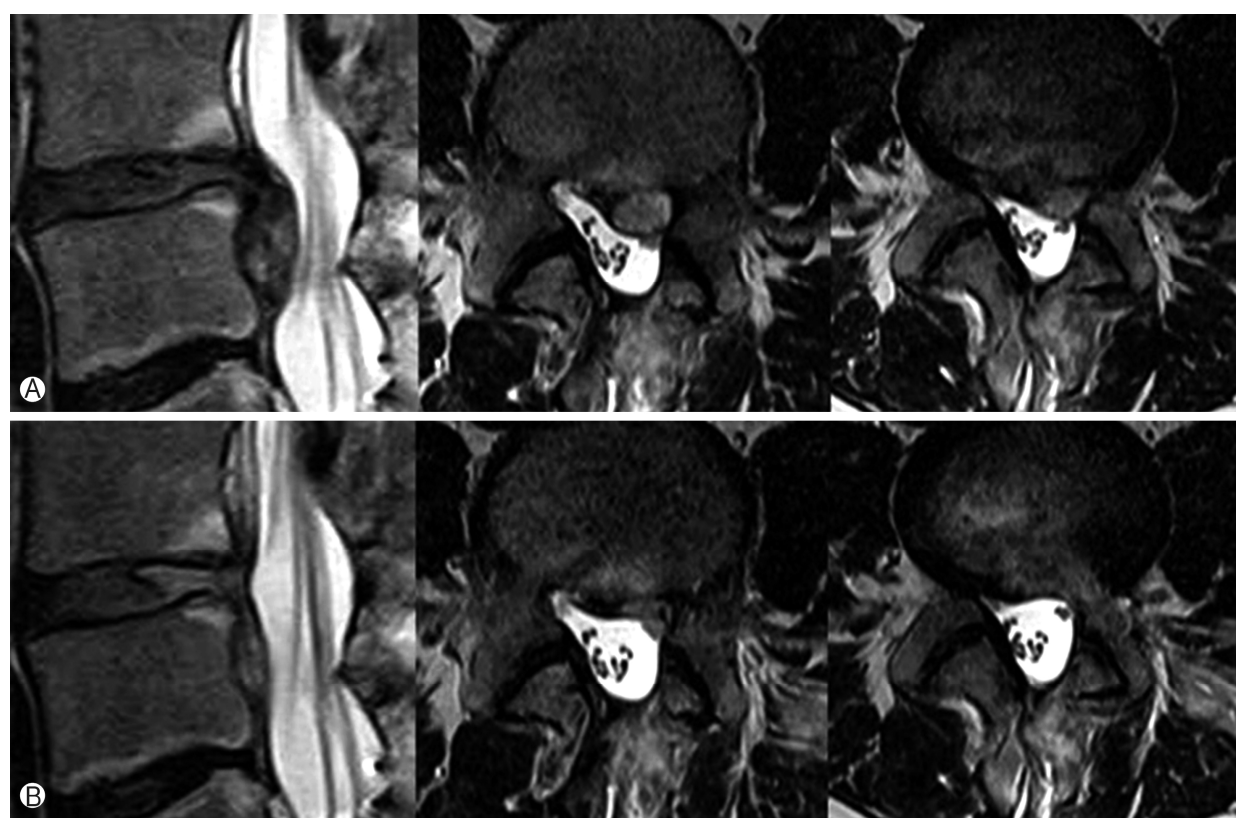

Fig. 2. Thirty-seven years old male with highly inferior migrated $L 3-4$ disc with left-sided foot drop. (A) Preoperative MRI, (B) Postoperative MRI 24 hours after suprapedicular circumferential opening technique to remove inferior migrated disc fragment. 
cal limitation for endoscope insertion, and there are two main surgical approaches: interlaminar and posterolateral. The endoscope insertion for posterolateral approaches is a blind procedure, but it can be safely achieved via the Kambin's triangle ${ }^{27)}$. During the interlaminar approach, structural damage includes not only ligamentum flavum but also paraspinal muscle and bony structures. Through structural preservation PEILD ${ }^{28)}$, it is possible to avoid this damage (Fig. 3). On the other hand, the insertion of the cannula for the interlaminar approach is performed under endoscopic visualization, but it is impossible to completely avoid direct retraction of the nerve root and/or dural sac by operative instruments. From previous experience with an open, microscopic, or microendoscopic discectomy, we recognize that some extent of transient retraction of the nerve root is acceptable. Nevertheless, we have to minimize the retraction as much as possible. In the meantime, the importance of the interlaminar approach has not been recognized as it is focused on solving some problems that the transforaminal approach cannot solve. However, the importance of the interlaminar approach is reappearing, as it is the basic approach for percutaneous endoscopic decompression.

\section{Goals of Application of Percutaneous Endoscopic Spine Surgery in the Degenerative Spinal Disease}

However, it is important to be aware of the fundamental purpose of percutaneous endoscopic spine surgery. In this respect, the pathophysiology of disc herniation and spinal stenosis and the concerns about the purpose of treatment according to each pathophysiology will provide a basis for this. In other words, the main pathology of lumbar disc herniation is nerve root compression ${ }^{29)}$ and it is considered that the most effective treatment can be achieved if it is effectively removed without any other peripheral damage. However, the pathologic hypertrophied ligaments, bony structures and vascular condition around the neural structure is the main cause of spinal stenosis ${ }^{30}$ so it is necessary to effectively remove the surrounding pathologic anatomical structures while maintaining sufficient spinal stability (Fig. 4).

The first goal to perform percutaneous endoscopic spine surgery is structural preservation procedure, preservation of functional segment being the second goal and rehabilitation for normal return to life is the third goal.

\section{FUTURE OF THE PERCUTANEOUS ENDOSCOPIC SPINE SURGERY}

\section{Application in Patients with the Severe Neurological Deficit}

There is a limitation in the application of percutaneous endoscopy in patients with the severe neurological deficit. More surgical expertise and development in armamentarium is required to increase the spectrum of endoscopy in these patients (Figs. $5,6)$.
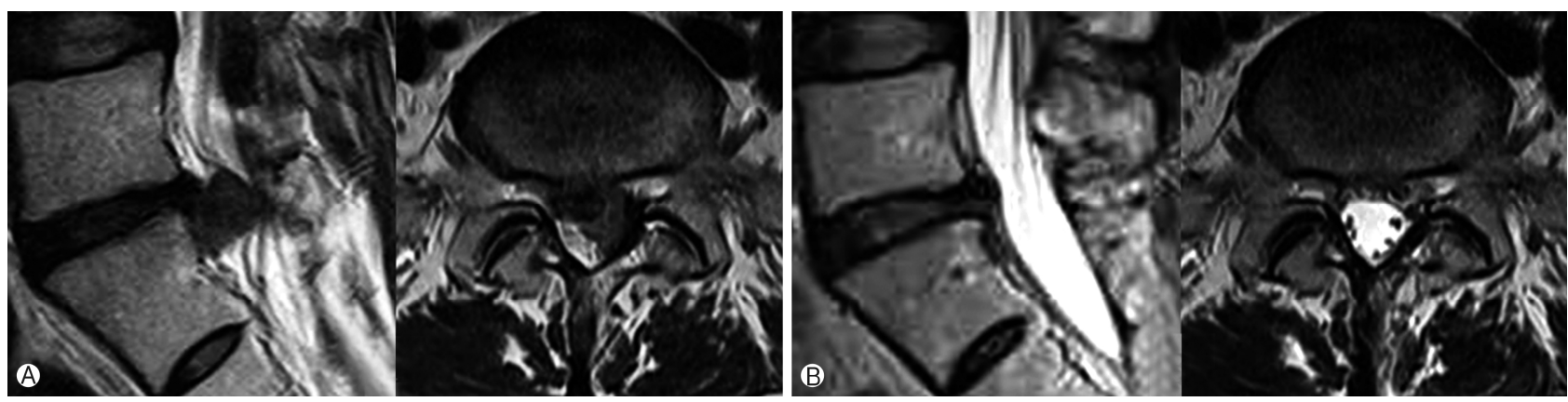

Fig. 3. Structural preservation PEILD for bilateral ruptured L5-S1 disc prolapse. (A) Preoperative MRI, (B) Postoperative MRI.
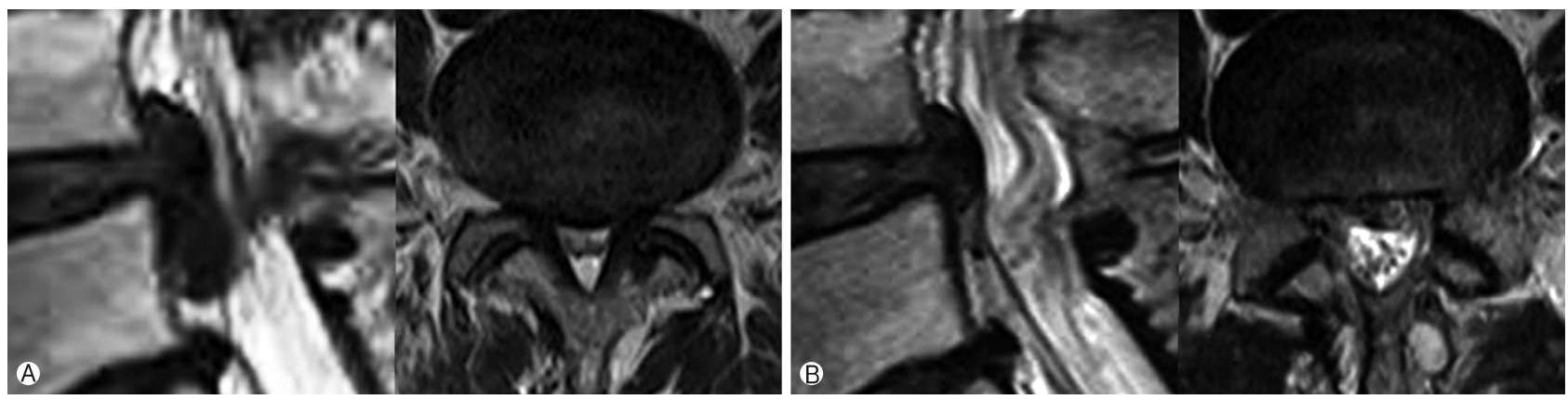

Fig. 4. Sixty-one years old female with back pain and Left leg pain (VAS 8) more than 6 months. With Left L5 weakness of grade 1. (A) Preoperative MRI, (B) Postoperative MRI. 


\section{Learning Curve}

The learning curve of the transforaminal approach is steep and easy to learn, while the learning curve of the interlaminar approach is flat and hard to master ${ }^{31)}$. Surgeons willing to spend the time and energy necessary to gain proficiency in endoscopy can expect to be rewarded through the benefits provided to their patients.
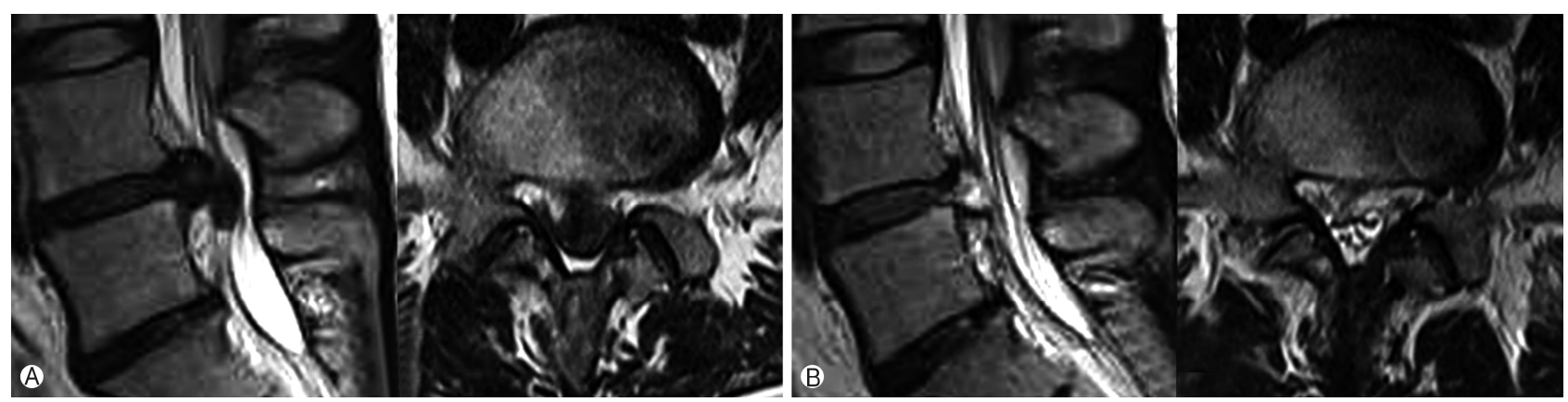

Fig. 5. Thirty-six years old female with left foot drop (L4: G0, L5: G0). (A) Preoperative MRI, (B) One day postoperative MRI after percutaneous endoscopic transforaminal lumbar discectomy. Patient motor weakness recovered completely 4 months after the operation.
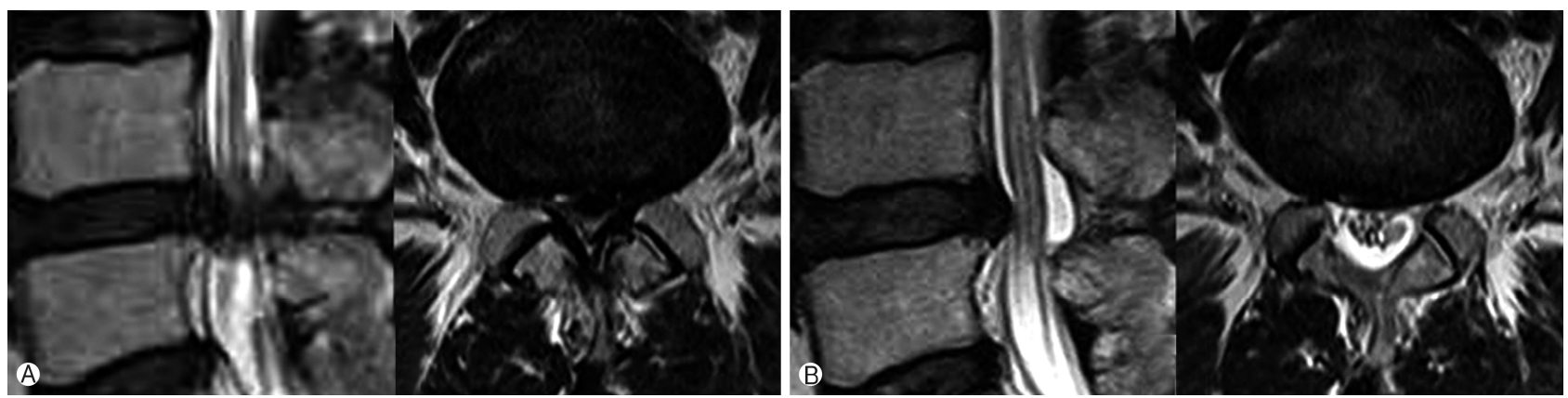

Fig. 6. Sixty-seven years old female with impending cauda equina syndrome. Back pain and severe both leg pain with aggravation since last 2 weeks and saddle anesthesia. (A) Preoperative MRI, (B) Postoperative MRI 24 hours after percutaneous endoscopic decompression.
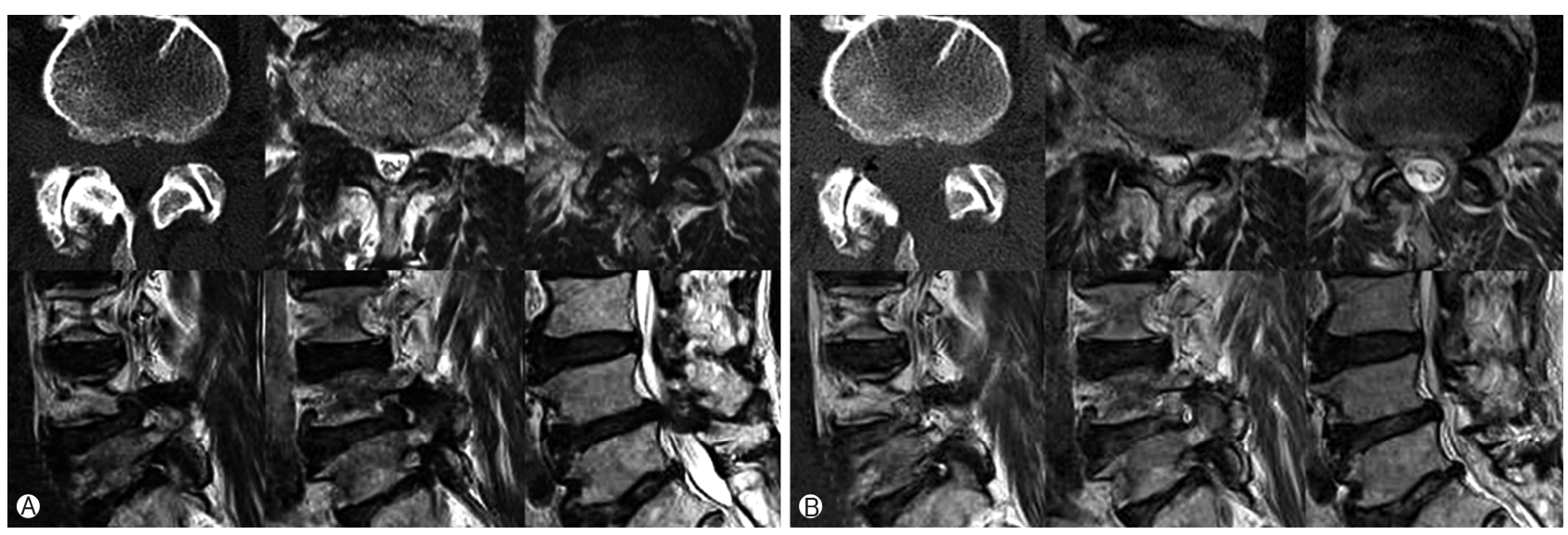

Fig. 7. Seventy-year-old male with low back pain, neurological intermittent claudication and both leg radiculopathy (R>L). Preoperative MRI (A) showing central spinal canal stenosis and foraminal to far lateral disc prolapse at right L4-5 level. Postoperative MRI (B) after endoscopic decompression and discectomy showing sufficient decompression without violation of facet joint as demonstrated on CT scan. 


\section{Safety Control}

In future endoscopic spine surgery is expected to be more widespread. It is planned to execute surgeries with better imaging systems and with 3D images ${ }^{32}$. At the same time, in order to enhance the sensitivity of the hand movements of surgeons and lessen the complication rates, it is expected that robotic surgery will be used in endoscopic spinal surgeries.

\section{Healthy Spine Life}

Endoscopic spine surgical technique has become more practical and standardized. Therefore, this technique may offer more reliable and reproducible results, especially for elderly or medically compromised patients to maintain healthy spine life (Fig. 7).

\section{CONCLUSION}

Percutaneous endoscopic spine surgery has evolved over the past several decades and is emerging as an important method of spine therapy for humans who want a healthy 100-year-old living in the future. However, it is true that the lack of necessary training courses and the development of equipment and technologies are still weak. In the future when these problems will be solved and percutaneous endoscopic spine surgery will be able to treat all problems of aging spine.

\section{ACKNOWLEDGEMENT}

We would like to acknowledge scientific team members Ms. Jae Eun Park and Mr. Kyeong-rae Kim for providing assistance in acquiring full text articles and managing digital works.

\section{REFERENCES}

1. Smith ZA, Fessler RG: Paradigm changes in spine surgery evolution of minimally invasive techniques. Nature Reviews Neurology 8(8):443-450, 2012

2. Yue JJ, Long W: Full endoscopic spinal surgery techniques: advancements, indications, and outcomes. International Journal of Spine Surgery 9, 2015

3. Ruetten S, Komp M, Merk H, Godolias G: Full-endoscopic interlaminar and transforaminal lumbar discectomy versus conventional microsurgical technique: a prospective, randomized, controlled study. Spine 33(9):931-939, 2008

4. Deukmedjian AJ, Cianciabella A, Cutright J, Deukmedjian A: Cervical Deuk Laser Disc Repair ${ }^{\circledR}$ : A novel, full-endoscopic surgical technique for the treatment of symptomatic cervical disc disease. Surgical Neurology International 3, 2012

5. Kim HS, Paudel B, Jang JS, Oh SH, Lee S, Park JE, et al: Percutaneous full endoscopic bilateral lumbar decompression of spinal stenosis through uniportal-contralateral approach: techniques and preliminary results. World Neurosurgery 103:201209, 2017

6. Nie H-F, Liu K-X: Endoscopic transforaminal thoracic foraminotomy and discectomy for the treatment of thoracic disc herni- ation. Minimally Invasive Surgery 2013, 2013

7. Ruetten S, Komp M, Merk H, Godolias G: A new full-endoscopic technique for cervical posterior foraminotomy in the treatment of lateral disc herniations using 6.9-mm endoscopes: prospective 2-year results of 87 patients. Minimally Invasive Neurosurgery 50(04):219-226, 2007

8. Choi G, Pophale CS, Patel B, Uniyal P: Endoscopic spine surgery. Journal of Korean Neurosurgical Society 60(5):485, 2017

9. Telfeian AE, Veeravagu A, Oyelese AA, Gokaslan ZL: A brief history of endoscopic spine surgery. Neurosurgical Focus 40(2): E2, 2016

10. Epstein NE: Spine surgery in geriatric patients: Sometimes unnecessary, too much, or too little. Surgical Neurology International 2, 2011

11. Transfeldt EE, Topp R, Mehbod AA, Winter RB: Surgical outcomes of decompression, decompression with limited fusion, and decompression with full curve fusion for degenerative scoliosis with radiculopathy. Spine 35(20):1872-1875, 2010

12. José-Antonio S-S, Baabor-Aqueveque M, Silva-Morales F: Philosophy and concepts of modern spine surgery. Advances in Minimally Invasive Surgery and Therapy for Spine and Nerves: Springer pp23-31, 2011

13. Kambin P, Gellman H: Percutaneous lateral discectomy of the lumbar spine a preliminary report. Clinical Orthopaedics and Related Research 174:127-132, 1983

14. Adsul N, Kim HS, Choi SH, Jang J-S, Jang I-T, Oh S-H: Acute bilateral isolated foot drop: changing the paradigm in management of degenerative spine surgery with percutaneous endoscopy. World Neurosurgery 110:319-322, 2018

15. Yeung A: Minimally invasive disc surgery with the yeung endoscopic spine system (YESS). Surgical Technology International 8:267-277, 1998

16. Yeung AT, Tsou PM: Posterolateral endoscopic excision for lumbar disc herniation: surgical technique, outcome, and complications in 307 consecutive cases. Spine 27(7):722-731, 2002

17. Hoogland T, Schubert M, Miklitz B, Ramirez A: Transforaminal posterolateral endoscopic discectomy with or without the combination of a low-dose chymopapain: a prospective randomized study in 280 consecutive cases. Spine 31(24):E890-E897, 2006

18. Ruetten S, Komp M, Godolias G: An extreme lateral access for the surgery of lumbar disc herniations inside the spinal canal using the full-endoscopic uniportal transforaminal approach - technique and prospective results of 463 patients. Spine 30(22): 2570-2578, 2005

19. Jang J-S, An S-H, Lee S-H: Transforaminal percutaneous endoscopic discectomy in the treatment of foraminal and extraforaminal lumbar disc herniations. Clinical Spine Surgery 19(5):338343, 2006

20. Min J-H, Kang S-H, Lee J-B, Cho T-H, Suh J-K, Rhyu I-J: Morphometric analysis of the working zone for endoscopic lumbar discectomy. Clinical Spine Surgery 18(2):132-135, 2005

21. Han J, Han I: Extreme lateral endoscopic transforaminal lumbar discectomy for lumbar disc herniation in young patients. The Nerve 2(2):26-32, 2016

22. Gore $S$, Yeung A: The "inside out" transforaminal technique to treat lumbar spinal pain in an awake and aware patient under local anesthesia: results and a review of the literature. International Journal of Spine Surgery 8, 2014 
23. Kim HS, Yudoyono F, Paudel B, Kim KJ, Jang JS, Choi JH, et al: Analysis of clinical results of three different routes of percutaneous endoscopic transforaminal lumbar discectomy for lumbar herniated disk. World Neurosurgery 103:442-448, 2017

24. Ahn Y, Oh H-K, Kim H, Lee S-H, Lee H-N: Percutaneous endoscopic lumbar foraminotomy: an advanced surgical technique and clinical outcomes. Neurosurgery 75(2):124-133, 2014

25. Choi G, Lee S-H, Raiturker PP, Lee S, Chae Y-S: Percutaneous endoscopic interlaminar discectomy for intracanalicular disc herniations at L5-S1 using a rigid working channel endoscope. Operative Neurosurgery 58(suppl_1):ONS-59-ONS-68, 2006

26. Ruetten S, Komp M, Godolias G: A new full-endoscopic technique for the interlaminar operation of lumbar disc herniations using 6-mm endoscopes: prospective 2-year results of 331 patients. Minimally Invasive Neurosurgery 49(02):80-87, 2006

27. Kambin P, Sampson S: Posterolateral percutaneous suction-excision of herniated lumbar intervertebral discs. Report of interim results. Clinical Orthopaedics and Related Research 207:37-43,
1986

28. Kim HS, Paudel B, Jang JS, Oh SH, Jang I-T: Percutaneous full endoscopic ligamentum flavum splitting interlaminar approach for removal of dorsally migrated lumbar disc herniation: a case report with technical note. Journal of Minimally Invasive Spine Surgery and Technique 2(1):35-38, 2017

29. Schoenfeld AJ, Weiner BK: Treatment of lumbar disc herniation: evidence-based practice. International Journal of General Medicine 3:209, 2010

30. Lee SY, Kim T-H, Oh JK, Lee SJ, Park MS: Lumbar stenosis: a recent update by review of literature. Asian Spine Journal 9(5):818-828, 2015

31. Hsu H-T, Chang S-J, Yang SS, Chai CL: Learning curve of fullendoscopic lumbar discectomy. European Spine Journal 22(4): 727-733, 2013

32. Middleton SD, Wagner R, Gibson JA: Multi-level spine endoscopy: A review of available evidence and case report. EFORT Open Reviews 2(7):317-323, 2017 